\title{
Moored observations of bottom-intensified motions in the deep Canada Basin, Arctic Ocean
}

\author{
by M.-L. Timmermans ${ }^{1}$, L. Rainville ${ }^{2}$, L. Thomas ${ }^{3}$ and A. Proshutinsky ${ }^{4}$
}

\begin{abstract}
In the deep Canada Basin, below the sill depth (about 2400 m) of the Alpha-Mendeleyev Ridge, potential temperature and salinity first increase with depth, then remain uniform from about $2600 \mathrm{~m}$ to the bottom (approximately $3500 \mathrm{~m}$ ). Year-long moored measurements of temperature, salinity and pressure in these deep and homogeneous bottom waters reveal significant vertical excursions with periods of about 50 days. The observed isopycnal displacements have amplitudes up to $100 \mathrm{~m}$ at the top boundary of the bottom layer; moored profiler measurements in the intermediate water column indicate that the amplitudes of these vertical displacements decay toward the surface over a scale of about $1000 \mathrm{~m}$. The subinertial excursions are consistent with a bottom-trapped topographic Rossby wave. Given the magnitude of the bottom slope in the vicinity of the mooring, the observed vertical velocities correspond to only weak (about $1 \mathrm{~cm} \mathrm{~s}^{-1}$ ) cross-slope horizontal velocities. The generation mechanism for the waves remains an open question.
\end{abstract}

\section{Introduction}

The deep and bottom waters in the Canadian Basin of the Arctic Ocean are effectively isolated from the shallow and intermediate-depth waters, having renewal timescales on the order of 450 years (Schlosser et al., 1997). In the Canada Basin (Fig. 1), below the 400 to 500-m depth warm core of the Atlantic Water layer, the potential temperature $\theta$ decreases with depth to a minimum temperature $\theta_{\min }$ near $2400 \mathrm{~m}$ (the approximate sill depth of the Alpha/Mendeleyev Ridge), increases with depth through an approximately $200 \mathrm{~m}$ thick layer, then remains uniform from about $2600 \mathrm{~m}$ to the bottom at $3500 \mathrm{~m}$ (Fig. 2). The well-mixed bottom layer implies that convective mixing is occurring as a consequence of geothermal heating (Timmermans et al., 2003). The salinity $S$ increases monotonically below the temperature minimum, then remains uniform in the bottom layer. A staircase structure, suggestive of double-diffusive convection, is observed between the temperature minimum and above the homogeneous bottom layer (Fig. 2). The staircase structure is

1. Department of Geology and Geophysics, Yale University, New Haven, Connecticut, 06511, U.S.A. email: mary-louise.timmermans@yale.edu

2. Applied Physics Laboratory, University of Washington, Seattle, Washington, 98105, U.S.A.

3. Environmental Earth System Science, Stanford University, Stanford, California, 94305, U.S.A.

4. Woods Hole Oceanographic Institution, Woods Hole, Massachusetts, 02543, U.S.A. 


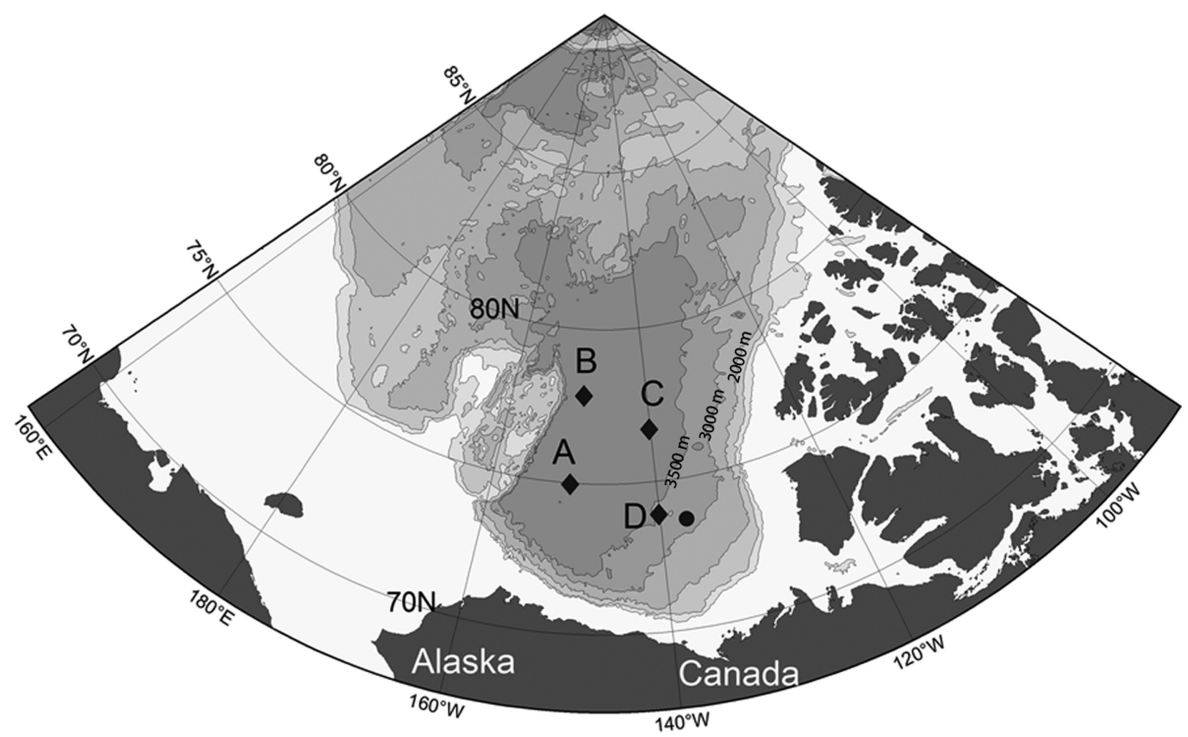

Figure 1. The Canada Basin in the Arctic Ocean. Isobaths have been plotted using the IBCAO grid (http://www.ibcao.org/). Beaufort Gyre Observing System (BGOS) mooring locations are marked by diamonds. The location of the 2002 pilot study (Timmermans et al., 2007) is marked by the black dot.

characterized by several 10 - to $40-\mathrm{m}$ thick mixed layers separated by $2-$ to $16-\mathrm{m}$ thick interfaces over which changes in potential temperature and salinity are only $\delta \theta \approx 0.003^{\circ} \mathrm{C}$ and $\delta S \approx 0.0007$ respectively (Timmermans et al., 2003; 2007). Timmermans et al. (2003) showed that the staircase structure is maintained by only a small fraction of the measured geothermal heat flux, and that most of the geothermal heat escapes in regions near the boundaries where the staircase is not observed. The staircase and bottom homogeneous layers extend laterally across the entire Canada Basin (Timmermans et al., 2003). It is likely that individual layers in the staircase extend horizontally for hundreds of kilometers across the basin as is the case for a double-diffusive staircase in the upper Arctic Ocean (Timmermans et al., 2008); data resolution, accuracy and coverage limitations prohibit confirmation of this for the deep staircase.

A 2002 pilot study, consisting of 50 days of high-resolution temperature measurements from a mooring at $73.5^{\circ} \mathrm{N}, 137^{\circ} \mathrm{W}$ (Fig. 1) with instruments spanning 2400 to $2650 \mathrm{~m}$, provided the first ever deep time series from the Canada Basin and revealed an unexpectedly active deep basin (i.e., it was much more dynamic than supposed previously based on the presence of the double-diffusive staircase). The measurements indicated vertical motions at the near-inertial (tidal) frequency on the order of 10 to $20 \mathrm{~m}$ and larger subinertial excursions of up to 50 meters (Timmermans et al., 2007). The pilot study motivated the observations presented here to determine the variability and source of the observed motion and and how it relates to processes in the intermediate depths. 

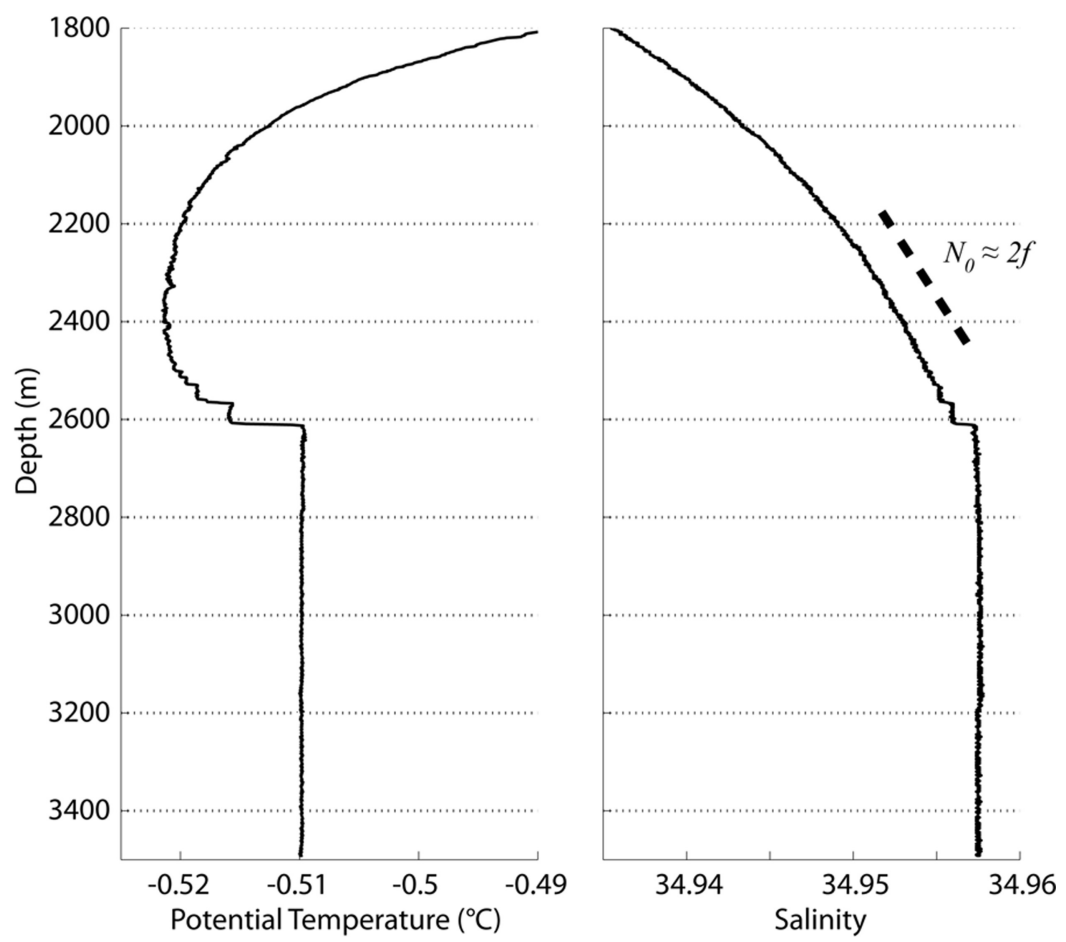

Figure 2. Profiles of potential temperature $(\theta$, left), and salinity $(S$, right $)$ in the Canada Basin in 2007 (site D, Fig. 1). The dashed sloped line in the right panel indicates stratification corresponding to $N_{0} \approx 2 f$ (note $\left.N_{0}^{2} \approx g \beta \overline{\partial S / \partial z}\right)$, where $f$ is the inertial frequency at $74^{\circ} \mathrm{N}(f=1.40 \times$ $\left.10^{-4} \mathrm{rad} \mathrm{s}^{-1}\right)$.

Here, we investigate temperature and salinity time-series measurements from instruments deployed on a bottom-anchored mooring at $74^{\circ} \mathrm{N}, 140^{\circ} \mathrm{W}$ (site D, Fig. 1) in 2007-2008 in the Canada Basin, and spanning the staircase overlying the bottom layer. The observations reveal isopycnal excursions with amplitudes that decrease with height above the seafloor, which slopes gently to the southeast in the vicinity of the mooring site. Bottom topography has been shown to be very important in the generation of low-frequency waves, even for small changes in bottom slope (Veronis, 1966). Topographic Rossby waves are subinertial motions induced by flow over sloping topography and subsequent stretching or compression of fluid columns (conservation of potential vorticity requires that when a fluid column is compressed it attains anticyclonic relative vorticity, and vice-versa). The theory for a bottom-trapped subinertial signal in the stratified ocean over a sloping seafloor in the absence of lateral boundaries was developed by Rhines (1970) (there is also an extensive literature on bottomtrapped waves in the presence of a coastline, e.g. Brink (1991)). Rhines (1970) derived an analytic dispersion relation for a topographic Rossby wave that propagates along the slope in a uniformly stratified ocean (a uniform buoyancy frequency $N$ over the water column). 
Reid and Wang (2004) extended the analysis of Rhines by deriving a topographic Rossby wave dispersion relation for an ocean having an exponential buoyancy frequency profile. Observational evidence for topographic Rossby waves has been examined extensively in the North Atlantic (e.g. Thompson and Luyten, 1976; Thompson, 1977; Pickart, 1995; Hogg, 2000) and in the deep basin of the Gulf of Mexico (e.g. Hamilton, 1990; 2009); in these cases, such waves have been shown to substantially modify deep-basin dynamics. Here we demonstrate the consistency of the data with a topographic Rossby wave propagating in the deep Canada Basin.

The paper is organized as follows. Section 2 presents the mooring observations and a description of the methods used to analyze motions in the deep water column. In Section 3, a topographic Rossby wave dispersion relation is derived that applies to an ocean having a uniform stratification above a homogeneous bottom layer (as in the deep Canada Basin). We further demonstrate how the observed isopycnal displacements are consistent with a topographic Rossby wave. Other possible mechanisms for the observed deep activity are discussed in Section 4, and results are summarized in Section 5.

\section{Observations and methods}

Beginning in summer 2003, an extensive field program has been conducted in the Canada Basin: the Beaufort Gyre Observing System (BGOS; www.whoi.edu/beaufortgyre) in order to document changes in sea ice and ocean parameters in the Beaufort Gyre (BG) centered in the Canada Basin (see Proshutinsky et al., 2009). Four moorings (A,B,C,D, Fig. 1) consist of a number of instruments that have provided time series of temperature, salinity, currents, sea-ice draft, and bottom pressure from the BG. Each mooring includes a McLane Moored Profiler (MMP) to record current and hydrographic profiles from about 50 to $2000 \mathrm{~m}$ (two profiles per 27 hours). Hydrographic measurements carried out every August/September from the CCGS Louis S. St-Laurent at approximately 30 sites in the BG are an integral component of the BGOS program (CTD profiles shown here are from these shipboard surveys). In 2007-2008, mooring D also supported instruments deeper than $2000 \mathrm{~m}$ to measure the evolution of temperature, salinity and pressure in the bottom layer and overlying staircase structure. We focus here on these measurements.

The instruments on the deep section of mooring D consisted of 12 temperature sensors (TR-1050 RBR Ltd, referred to here as TR1-TR12), two temperature, conductivity and pressure recorders (XR-420CTD RBR Ltd, referred to as TXR1 and TXR2), and two temperature, conductivity recorders (an XR-420CT [TXR] and a Sea-Bird SBE37). The instruments were positioned to span the deep staircase structure (Fig. 3). TR1 - TR12 sampled temperature every minute, TXR1, TXR2 and TXR sampled every 3 minutes, and the SBE37 sampled every 15 minutes between 24 August 2007 and 13 August 2008. Sensors were calibrated using a CTD profile taken 13 hours prior to the first mooring measurements. Corrections were less than $0.001^{\circ} \mathrm{C}$ for each of TR1 - TR12 and less than $0.005^{\circ} \mathrm{C}$ for the other instruments. Comparison to the post-deployment CTD cast indicated accuracies better 

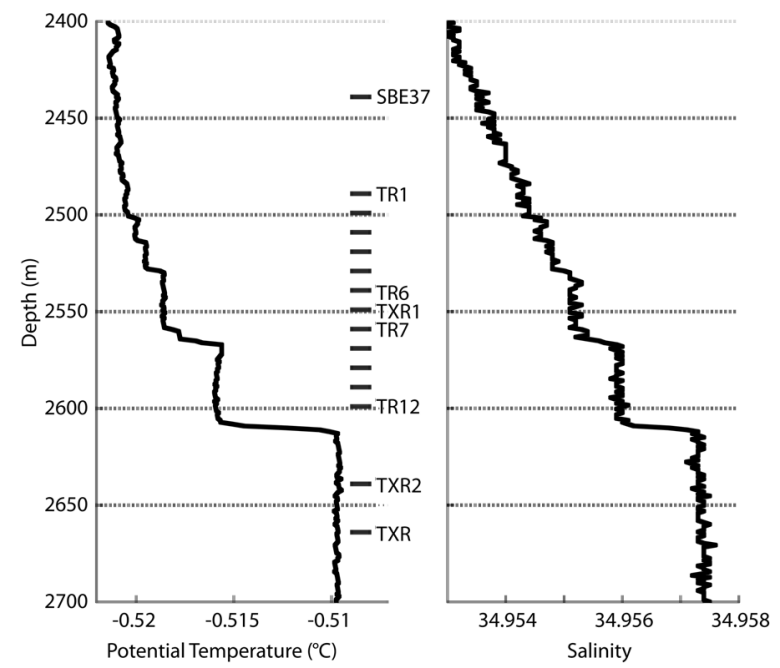

Figure 3. Potential temperature (left) and salinity (right) profiles taken at site D on 8/23/2007 prior to deployment of the mooring. Depths spanned by the instruments on the deep section of mooring D are shown (from shallowest to deepest: SBE37, TR1, TR2, TR3, TR4, TR5, TR6, TXR1, TR7, TR8, TR9, TR10, TR11, TR12, TXR2, TXR).

than $0.002^{\circ} \mathrm{C}$. Hence, it is reasonable to assume no drift of the sensors over the year-long deployment.

The conductivity sensors on both TXR1 and TXR2 showed erratic behavior that could not be corrected for reliable observations and so are not included here. No corrections were made to the pressure sensors on these instruments which have accuracies better than $3 \mathrm{dbar}$ and resolutions of $0.06 \mathrm{dbar}$. The SBE37 salinity differed from the start CTD value by 0.01 and was corrected accordingly; resolution is 0.0002 . After correction using the start CTD value, comparison to the post deployment CTD profile indicated sensor accuracy about 0.005. Note that all instruments were re-deployed in September 2008 with only enough time between deployments to download the data. Hence, post-deployment calibrations were not made.

Figure 4 shows the year-long time series of potential temperature and salinity from deep $\mathrm{D}$ instruments (only three instruments are shown for clarity). Discrete jumps in temperature are apparent in the vicinity of the staircase (about $-0.52^{\circ} \mathrm{C}$ to $-0.51{ }^{\circ} \mathrm{C}$ ). Linear fits indicate no significant trend in temperature of either the bottom layer (TXR) or any other location sampled during the deployment up to the potential temperature minimum $\left(\theta_{\min }\right)$. Trends on the order of $0.001{ }^{\circ} \mathrm{C}$ per year would be detectable, so that the data are consistent with a steady-state balance between geothermal heat flux into the bottom layer, and diffusive heat flux at the basin boundaries and through the staircase (Timmermans et al., 2003).

Temperature fluctuations over a range of frequencies are apparent in the time seriesmeasurements. Enhanced energy levels are visible in a peak corresponding to the 


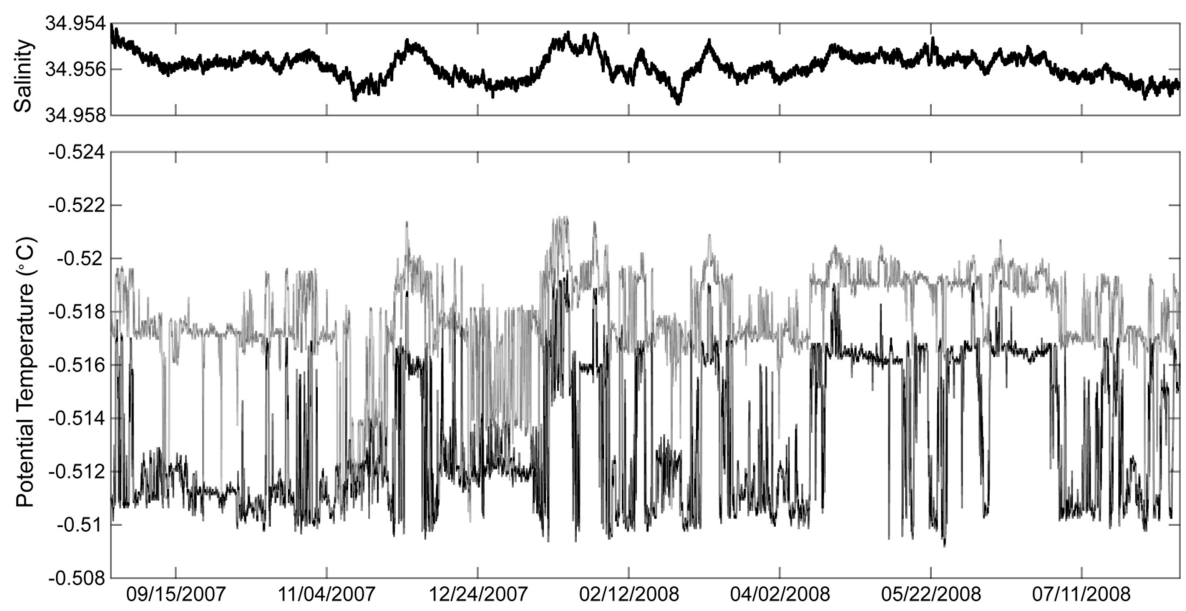

Figure 4. Salinity time series from the SBE37 at $2439 \mathrm{~m}$ (top), and potential temperature time series from two deep instruments on mooring D (TR12, black and TR9, grey).

semi-diurnal tidal frequency $\left(M_{2}=1.41 \times 10^{-4} \mathrm{rad} \mathrm{s}^{-1}\right)$ and the inertial frequency, $f=1.40 \times 10^{-4} \mathrm{rad} \mathrm{s}^{-1}$ (Fig. 5). Note that these are not separable; both baroclinic motions are intermittent and will also show up as finite frequency bands due to interactions with the varying background. Pressure sensor frequency spectra (from TXR1 and TXR2, not shown) indicate the same peak plus a diurnal peak due to the barotropic tide. Vertical motions of the water column (on the order of $10 \mathrm{~m}$ ) cannot be inferred from sensors positioned where there are no sharp gradients between mixed layers in the staircase and/or where vertical temperature gradients are weak (in the bottom layer and above the staircase around $\theta_{\min }$ ); an absence of fluctuations does not necessarily imply an absence of movement. In the next section we show how a background of much longer period (subinertial frequency) motions is also evident.

\section{a. Sub-inertial motion at site $D$}

$i$. Deep water column. Changes in water properties measured by the deep moored instruments could arise from mooring motion, vertical motion of the water column, or advection of horizontal gradients. Pressure time series from TXR1 and TXR2 allow us to rule out mooring motion as contributing significantly to temperature fluctuations (Fig. 6). Over the year-long record, the only mooring motions of note are two pressure excursions in FebruaryMarch 2008 that mark pull-down of the mooring by less than $2 \mathrm{~m}$ at the depth of the staircase by a passing eddy (or eddies). This is a Pacific Water eddy centered around $150 \mathrm{~m}$ depth, as measured by the MMP on the upper section of mooring D.

To analyze the deep motions, we begin by assuming that temperature signals are due to vertical motion of the water column. Note that CTD profiles of potential temperature and salinity at site D between 2003 and 2008 indicate that the staircase structure varies in depth; 


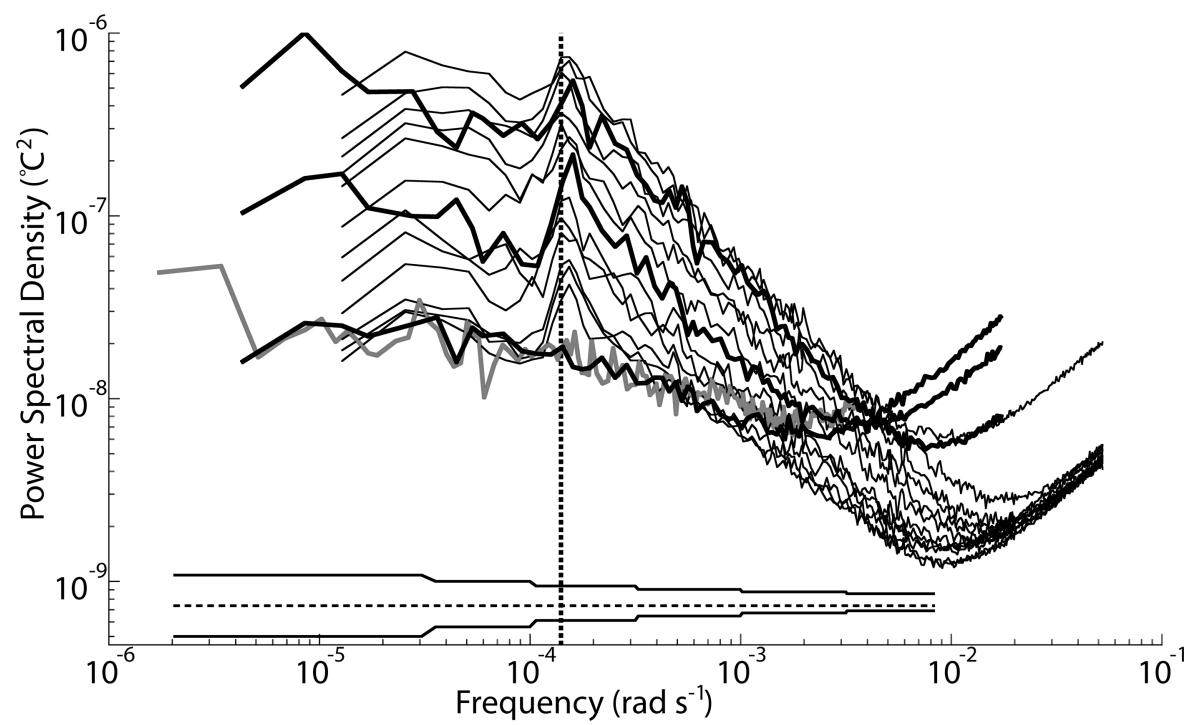

Figure 5. Frequency spectra of temperature time series on mooring D. SBE37 (grey), TXR, TXR2, TXR1 (thick black, lowest to highest), TR12-TR1 (thin black, lowest to highest, with TR11 having a higher noise floor than the others). The vertical dotted line is $f, M_{2}$ and confidence limits (95\%) for TR12-TR1 are shown below the spectra. Note that absence of motion cannot be implied necessarily by the absence of temperature fluctuations recorded by sensors positioned where there are no sharp gradients between mixed layers in the staircase and/or where vertical temperature gradients are weak.

vertical variability on the order of $50 \mathrm{~m}$ is evident between years (Fig. 7). We further assume that movement of the staircase unit is uniform over the vertical span of the deep instruments on mooring D. A vertical displacement time series can then be inferred by matching the CTD profile of potential temperature $\theta(z)$ (Fig. 3) to the deep time series measurements at site D. At each sample time, the vertical displacement $\eta$ is obtained by minimizing the variance between the temperature data (from TR1-TR12, TXR1, TXR2, TXR and SBE37) and displaced CTD profile $[\theta(z+\eta)]$ (Fig. 6). A warm temperature excursion, for example, implies that water has risen from below the instruments and $\eta>0$. The displacement time series $\eta$ is found to agree in general with a displacement time series obtained by matching the CTD profile of salinity to the SBE37 salinity time series. This indicates that stable intrusions or density overturns do not introduce significant errors using this approach and displacement of isopycnal surfaces (heave) takes place with little or no change in water mass properties.

Advection of horizontal gradients can also contribute to variability measured by the instruments on the mooring. Horizontal gradients in temperature and salinity in the vicinity

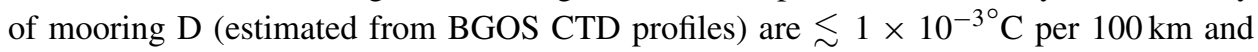
$\lesssim 3 \times 10^{-4}$ per $100 \mathrm{~km}$ respectively. An inertial current at this latitude (with 12 hour period) 


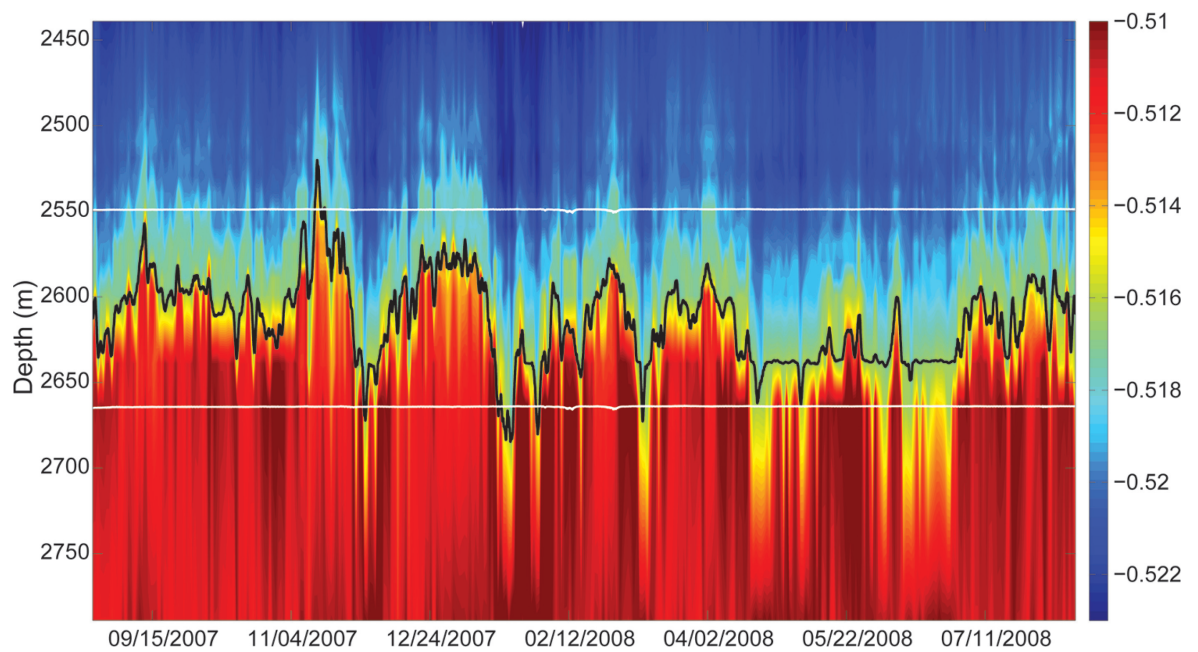

Figure 6. Depth-time section of potential temperature from all deep instruments on mooring D. The inferred vertical displacement $\eta$ (offset by $2600 \mathrm{~m}$ ) is shown by the black line (the moving average over the $M_{2}$ tidal cycle). Depth-time series from TX1 and TX2 (calculated from pressure) are shown by the gray lines; note that mooring motion is negligible compared to vertical displacements.
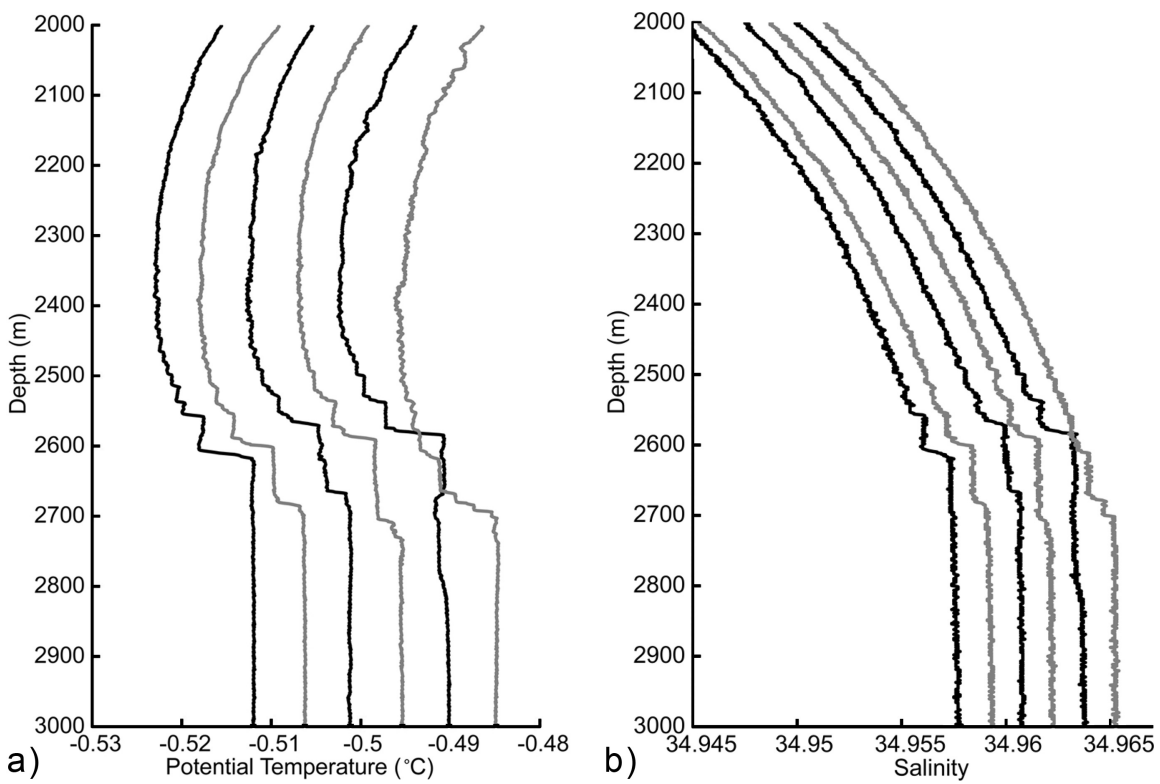

Figure 7. (a) Potential temperature at mooring site D from August 2003 (left profile) to August 2008 (right profile). Profiles are offset by $0.005^{\circ} \mathrm{C}$. (b) Corresponding salinity profiles offset by 0.0015 . 
of magnitude $5 \mathrm{~cm} \mathrm{~s}^{-1}$ would have a horizontal excursion of about $2 \mathrm{~km}$. Advection of horizontal gradients over these distances would yield negligible temperature and salinity signals (i.e. negligible bias in the calculation of $\eta$ ). Timmermans et al. (2007) note that a seiche (a free oscillation of the water column triggered by a passing atmospheric disturbance) in the main pycnocline can lead to lateral motion at the depth of the staircase. A lowest order seiche could result in about 4-km lateral motion at the depth of the staircase in one seiche period (about 15 days). Based on the horizontal gradients in temperature and salinity, this lateral advection would result in negligible temperature and salinity fluctuations and spurious vertical excursions $\lesssim 1 \mathrm{~m}$, much less than the estimated displacements.

While there is some variability (merging or splitting of layers) within the staircase itself, this does not introduce significant error into the estimate of $\eta$ since the signal is typically measured by only 1 or 2 of the 16 instruments on the deep portion of mooring D (Fig. 8). Due to limited vertical resolution, it is unclear whether there are occurrences of split layers that appear to form and re-merge in hours, or whether the observations are indicating thickness changes in layers. Split layers are commonly observed in oceanographic profiles from other regions (Kelley et al., 2003). Kelley (1988) suggested that staircase layer thickness is controlled by layer splitting where new layers are formed from existing interfaces. It may be that split layers form and re-merge during times of significant motion. There is temporal temperature variability of the layers (also likely caused by merging and splitting), although never exceeding the difference in temperature between two adjacent layers for the deepest three layers and this does not contribute significant error to $\eta$.

The vertical displacement time series indicates that in addition to motion in the internalwave frequency band (e.g. Fig. 5), vertical excursions on the order of tens of meters over many days are also evident (Fig. 6). We restrict our attention in this paper to this subinertial variability. A wavelet analysis enables us to examine the frequency distribution over time of the more intermittent subinertial motion. A Mortlet wavelet transform (e.g. Torrence and Compo, 1998) of daily-averaged vertical displacement indicates a peak wave period of around 50 days (Fig. 9).

ii. Intermediate water column. The MMP on the upper section of mooring D allows us to assess the relationship between motions at depth and those in the intermediate water column (Fig. 10). The correlation between isopycnal displacements at intermediate depths and those in the deep water is clear (Note that the MMP stopped profiling to the full programmed depth $(1970 \mathrm{~m})$ after April 18, 2008.) Also apparent is that the motions increase in amplitude with depth. In the next section, we investigate possible mechanisms for the bottom-intensified subinertial signal.

\section{Results}

In the vicinity of mooring D, IBCAO bathymetry indicates the bottom sloping gently up toward the southeast with a gradient of order $100 \mathrm{~m}$ in $20 \mathrm{~km}$. Near the bottom, an up-slope 

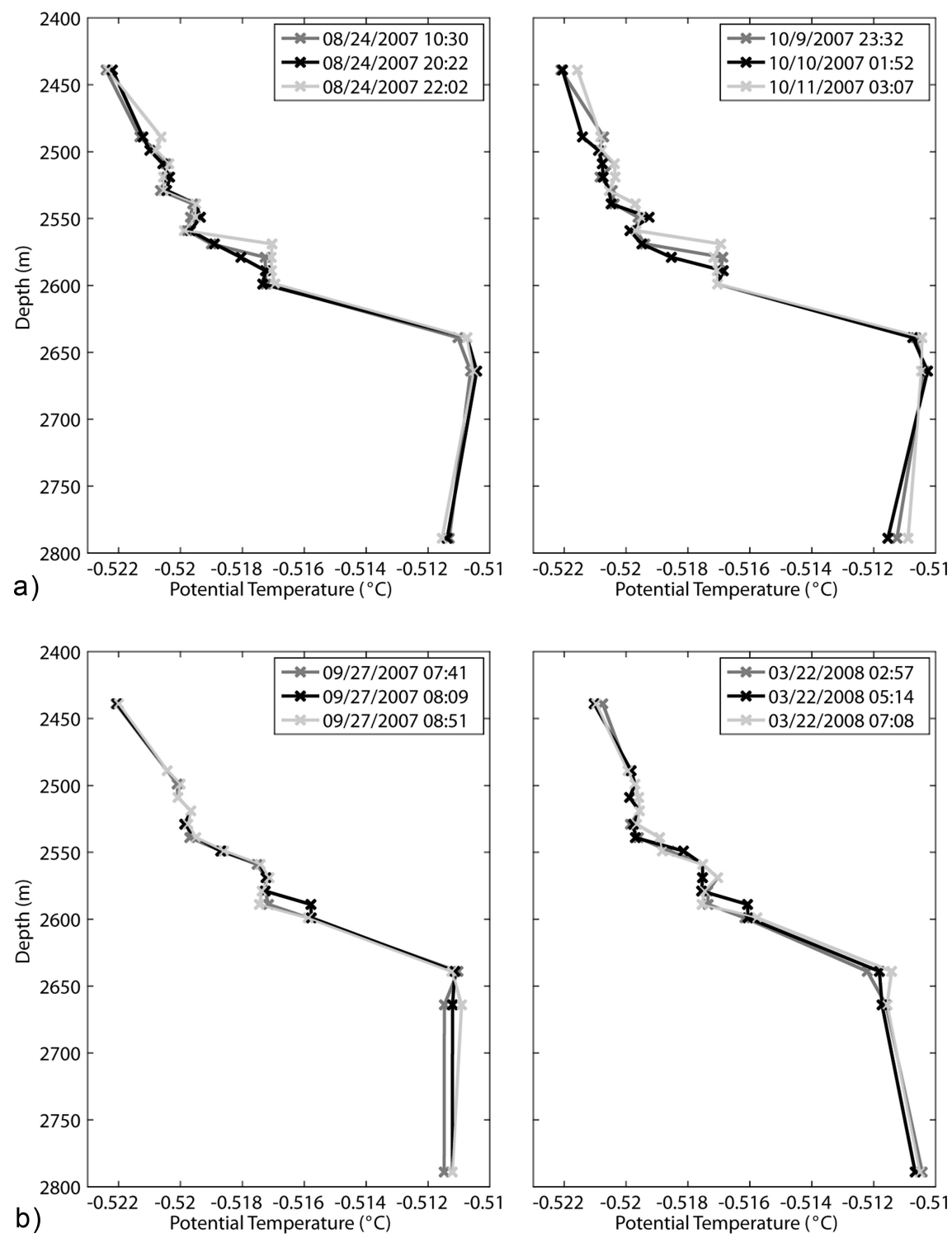

Figure 8. Potential temperature profiles through the staircase at site D indicating what may be either splitting and merging or layer thickness changes.

velocity must be accompanied by an upward vertical velocity. Given the magnitude of the bottom slope in the vicinity of the mooring, the observed vertical velocities (vertical excursions of about $100 \mathrm{~m}$ in about 20 days) correspond to cross-slope horizontal velocities of about $1 \mathrm{~cm} \mathrm{~s}^{-1}$. It is reasonable to speculate that the observed periodic isopycnal 

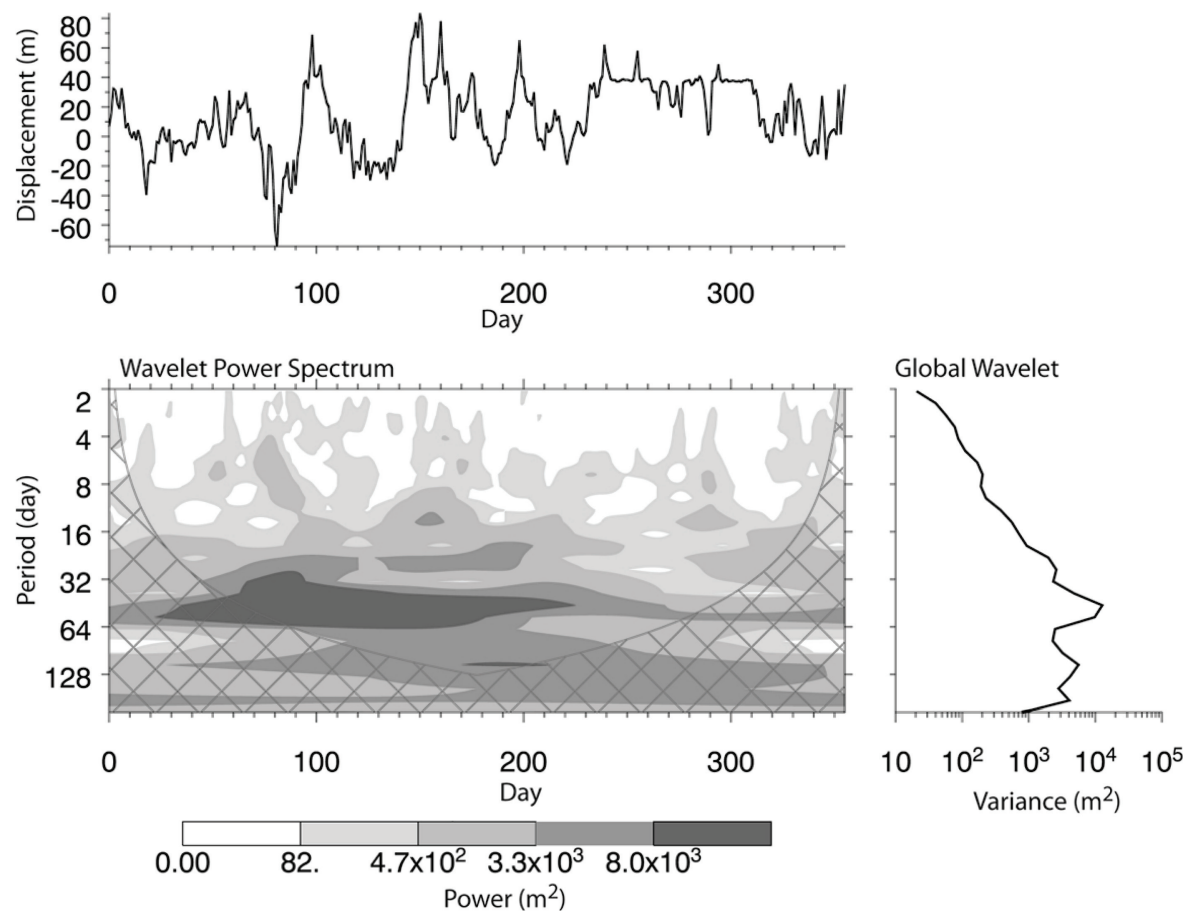

Figure 9. Top Panel: Daily-averaged time series of vertical displacement $\eta$. Bottom Left: Mortlet wavelet transform of daily-averaged vertical displacement $\eta$. The dashed cone-of-influence indicates the maximum period of useful information at that particular time. Periods longer than this are subject to edge effects. Bottom Right: The global wavelet power spectrum indicating a peak wave period of around 50 days.

displacements are associated with a topographic Rossby wave propagating at subinertial frequencies (Rhines, 1970). To test this, we derive the appropriate dispersion relation, given the stratification shown in Figure 2.

Assume simple bottom topography in which the depth decreases linearly in the $y$ direction (we consider an $f$-plane and take $y$ to be the cross-slope coordinate with $x$ directed along-slope) with bottom slope angle $\nu$. Using the hydrostatic approximation, the governing equations are

$$
\begin{aligned}
\frac{\partial u}{\partial t}-f v & =-\frac{1}{\rho_{0}} \frac{\partial p}{\partial x} \\
\frac{\partial v}{\partial t}+f u & =-\frac{1}{\rho_{0}} \frac{\partial p}{\partial y} \\
0 & =-\frac{1}{\rho_{0}} \frac{\partial p}{\partial z}+b
\end{aligned}
$$




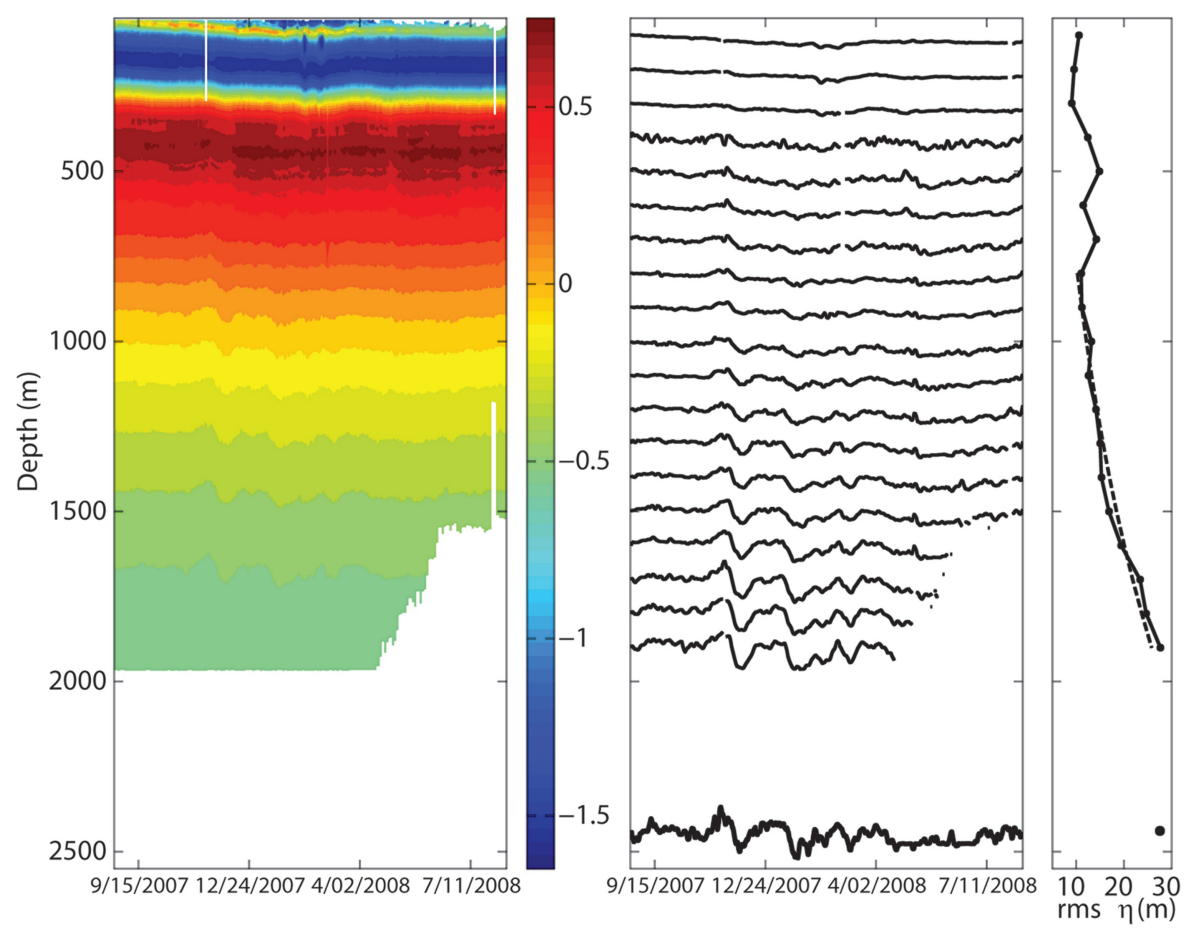

Figure 10. Left: Depth-time section of potential temperature $\left({ }^{\circ} \mathrm{C}\right)$ from the shallow MMP on mooring D. Middle: Depth-time section showing isopycnals and the inferred vertical displacement $\eta$ (the thick line near the bottom of the plot) offset by $2439 \mathrm{~m}$, the depth of SBE37 (moving averages over 1 day). Isopycnals were selected to have equal depth spacing $(100 \mathrm{~m})$ at the start of the record. Right: Vertical profile of rms vertical displacement calculated from the isopycnals shown in the middle panel and from $\eta$, where the time-series have been low-pass filtered to suppress periods shorter than 10 days. The dashed line shows the exponential fit used to estimate the vertical decay scale of the wave.

$$
\begin{array}{r}
\frac{\partial b}{\partial t}+w N^{2}=0 \\
\frac{\partial u}{\partial x}+\frac{\partial v}{\partial y}+\frac{\partial w}{\partial z}=0
\end{array}
$$

where $\mathbf{u}=(u, v, w), b=-g(\rho-\bar{\rho}) / \rho_{0}$, and $p$ are the wave velocity, buoyancy and pressure and $N^{2}=-g / \rho_{0}(\partial \bar{\rho} / \partial z)$ is the square of the buoyancy frequency associated with the background density field, $\bar{\rho}$ ( $\rho_{0}$ is a reference density). The governing equations can be reduced to a single equation for the vertical velocity, which in the limit of subinertial motions, $\omega^{2} / f^{2} \ll 1$, is

$$
f^{2} \frac{\partial^{2} w}{\partial z^{2}}+N^{2}\left(\frac{\partial^{2} w}{\partial x^{2}}+\frac{\partial^{2} w}{\partial y^{2}}\right)=0 .
$$


The velocity must satisfy no normal flow at the bottom boundary,

$$
w=v \tan \nu \approx v \nu \quad \text { at } \quad z=-H,
$$

for water depth $H$ and $z$ is positive upward (normal to horizontal). Seeking solutions for $v$ and $w$ of the form

$$
\left(\begin{array}{c}
v \\
w
\end{array}\right)=\operatorname{Re}\left\{\left(\begin{array}{c}
V(z) \\
W(z)
\end{array}\right) \exp [i(k x+l y-\omega t)]\right\},
$$

we find $d^{2} W / d z^{2}=0$ in the bottom homogeneous $(N=0)$ layer $\left(-H<z<-H_{T}\right.$, where $z=-H_{T}$ is the top boundary of the bottom layer). Assume $W\left(=W_{0}\right)$ is constant in this layer, so that (7) yields

$$
W_{0}=V(z=-H) \nu .
$$

The wave's buoyancy $b$ is zero in the bottom layer since $N^{2}=0$, and the thermal wind relation,

$$
f \partial v / \partial z=\partial b / \partial x
$$

leads to $V=V_{0}$, a constant in the bottom layer. Hence, $W_{0}=V_{0} \nu$. Now, let us approximate the stratification overlying the bottom layer $\left(z \geq-H_{T}\right)$ by $N^{2}=N_{0}^{2}$, constant. Then, the solution for the vertical velocity for $z \geq-H_{T}$, from (6), is

$$
w=\operatorname{Re}\left\{V_{0} \nu \exp \left[-\gamma\left(z+H_{T}\right)\right] \exp [i(k x+l y-\omega t)]\right\},
$$

where $\gamma=N_{0} k_{H} / f$ and $k_{H}^{2}=k^{2}+l^{2}$. The wave decays above the bottom layer, with vertical decay scale $1 / \gamma=f L /\left(N_{0} 2 \pi\right)$, where $L$ is the horizontal scale of the wave. We estimate $\gamma \approx 0.001 \mathrm{~m}^{-1}$ from the vertical decay scale obtained by fitting an exponential to a vertical profile of the rms isopycnal displacement (Fig. 10). Hence, the horizontal wavelength of the wave is small, $L \approx 4 \pi / \gamma \approx 12 \mathrm{~km}$.

It is straightforward to derive the dispersion relation. From (4), it follows that for $z \geq$ $-H_{T}, b=\operatorname{Re}\left\{B_{0} \exp \left[-\gamma\left(z+H_{T}\right)\right] \exp [i(k x+l y-\omega t)]\right\}$, which, upon substitution gives $B_{0}=-i N_{0}^{2} V_{0} v / \omega$. Substituting the solutions for $b$ and $v$ (Eq. (8)) into (10) yields

$$
\frac{d V}{d z}=\frac{N_{0}^{2}}{\omega f} \nu k V_{0} \exp \left[-\gamma\left(z+H_{T}\right)\right]
$$

Recall $V=V_{0}$ (constant) in the bottom homogeneous layer, so that $V\left(z=-H_{T}\right)=V_{0}$ and $V(z)=V_{0} \exp \left[-\gamma\left(z+H_{T}\right)\right]$. Substituting $V(z)$ into (12) yields the simple dispersion relation

$$
\omega=-N_{0} \nu \frac{k}{k_{H}} .
$$

The wavevector and energy propagation are in directions to the left of the direction of decreasing water depth $(k<0)$, with the group velocity (normal to the wavevector) given by

$$
\mathbf{C}_{\mathbf{g}}=\left(\frac{\partial \omega}{\partial k}, \frac{\partial \omega}{\partial l}\right)=\frac{N_{0} \nu}{k_{H}^{2}}\left(-l^{2}, k l\right)
$$


Observations are well inside the parameter space corresponding to bottom-trapped topographic Rossby waves. Conservative ranges for the bottom slope and stratification in the vicinity of mooring $\mathrm{D}$ are $v \approx 0.003-0.005$ and $N_{0} \approx 2 f-3 f$, respectively. Estimating the period of vertical isopycnal displacements to be between 45 to 55 days (Fig. 9), we find the wavevector satisfies $1 / 3 \lesssim k^{2} / k_{H}^{2} \lesssim 1$.

Bottom intensification, wave frequency and wavevector are consistent with bottomtrapped topographic Rossby waves. Future additional field data together with ray-tracing techniques (e.g. Pickart, 1995; Hamilton, 2009) using the observed bottom topography and stratification can be used to further constrain the direction of propagation and locate possible sources.

To our knowledge there have been no other observations of topographic Rossby waves in this region of the basin, although early numerical experiments by Galt (1973) point to the prevalence of topographic Rossby waves in the Arctic Ocean. Zakharchuk (2009) analyzed current measurements in the Chukchi Sea that indicated topographic Rossby waves with periods between 7 and 60 days that appear to be forced by local winds. Note also that Voinov and Zakharchuk (1999) show the presence of topograhic Rossby waves in the Laptev Sea.

\section{Discussion}

While we have made the case that the isopycnal displacements are likely associated with bottom-trapped topographic Rossby waves, alternative processes should be considered. The possibility remains, for example, that density intrusions cause subinertial excursions at site D. An intrusion in the water column shallower than the SBE37 (at $2439 \mathrm{~m}$ ) and deeper than the bottom depth of the profiling MMP (1970 m) would not be sampled. However, an intrusion in this portion of the water column would result in isopycnal displacements of the opposite sign above and below.

Significant intrusive activity between $\theta_{\min }$ and the top of the bottom layer can be ruled out by examining the temperature and salinity time series from the SBE37 at the top boundary of the staircase (Fig. 4). In the case of density intrusions, temperature and salinity fluctuations satisfy $\alpha \delta \theta=\beta \delta S$, where $\alpha=1.2 \times 10^{-4}{ }^{\circ} \mathrm{C}^{-1}$ is the coefficient of thermal expansion and $\beta=7.6 \times 10^{-4}$ is the coefficient of saline contraction. Hence, for intrusions, temperature fluctuations would be about six times larger than salinity fluctuations $(\beta / \alpha \approx 6)$, while they are observed to be about the same (recall further that the displacement time series $\eta$ is very similar to a time series obtained by matching the CTD profile of salinity to the SBE37 salinity time series).

We cannot rule out intrusive activity in the bottom layer as the cause of the observed motion. Although steady temperature changes in the bottom layer are negligible, temperature fluctuations are observed, and may be associated with intrusions. It may be that cool, fresh intrusions are indicated in the temperature time series (Fig. 11), in which the temperature change $\delta \theta \approx 0.001^{\circ} \mathrm{C}$ would be compensated by $\delta S \approx 0.0002$. Based on lateral temperature and salinity gradients in the vicinity of the mooring from the 2007 CTD data 

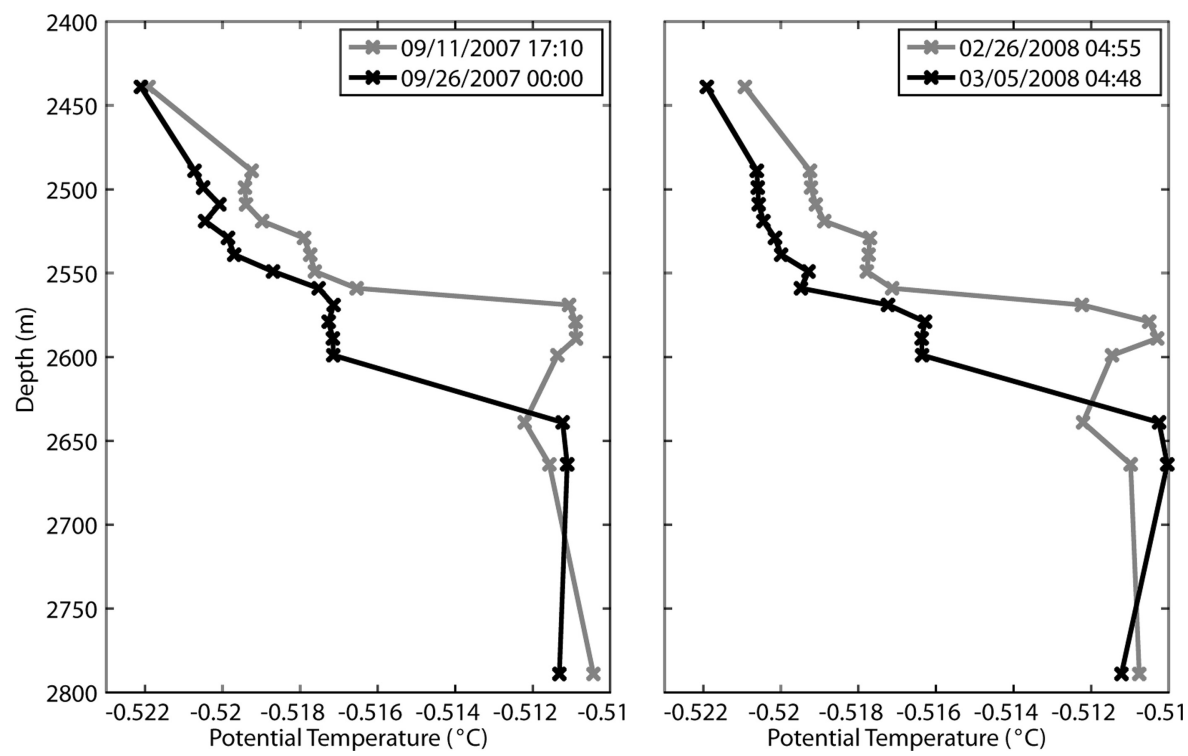

Figure 11. Potential temperature profiles through the staircase at site D showing what may be either cool fresh intrusions, density overturns in the bottom layer during times of large vertical displacement, or merging/splitting of staircase layers that cannot be resolved by the vertical spacing of the deep instruments. Note the staircase profiles shown here are skewed due to the positions of the instruments on the mooring.

around $2700 \mathrm{~m}$, a cool fresh intrusion coherent over less than $100 \mathrm{~km}$ could explain the measurements. It is conceivable that such an intrusion could induce shoaling of the staircase structure although in this case, we might expect potential temperature fluctuations in the bottom layer (at TXR) to be correlated with $\eta$; the two time series show no correlation. Intrusion scenarios cannot be tested without additional salinity information. The features described above could alternatively be density overturns driven by local mixing (that does not significantly impact $\eta$ ). In this case, the subinertial isopycnal motions may contribute to deep basin mixing, although without salinity information, this remains speculative.

Finally, it is possible that the observations may be associated with a basin-mode oscillation of the semi-enclosed Canada Basin. In this case, we expect coherent variability between deep motions at site $\mathrm{D}$ and $\mathrm{A}$; there is no apparent correlation of the subinertial variability between the two sites (MMPs operated simultaneously on both moorings between October 2007 and March 2008), suggesting a sub-basin scale process.

\section{Summary}

Temperature and salinity measurements reveal significant vertical motion in the vicinity of the deep staircase structure in the Canada Basin. Mooring motion, measured by pressure recorders, accounts for less than about $2 \%$ of the variance. MMP data from the intermediate 
water column indicate that the subinertial motions increase in amplitude with depth. Large subinertial excursions with rms displacements of about $30 \mathrm{~m}$ and a peak period of about 50 days are evident (variability at inertial frequencies $\left(f, M_{2}\right)$ is superimposed on the low frequency).

The 50-day period subinertial isopycnal displacements are consistent with bottomtrapped topographic Rossby waves. The motions are bottom-intensified with a vertical scale spanning about $1000 \mathrm{~m}$ from the bottom layer and a wavelength of about $12 \mathrm{~km}$. Observations require cross-slope velocities of about $1 \mathrm{~cm} \mathrm{~s}^{-1}$ near the bottom at site D. The generation mechanism for the observed waves remains an open question. The group velocity is to the left of the direction of decreasing water depth, which could imply that the waves originate from the southwest of site D. They may be generated, for example, by strong winter storm activity over the Beaufort Sea continental slope (e.g. Pickart et al., 2009) to the southwest of the mooring site. Another possibility is their generation by deep eddies, of a type that has been recorded at Beaufort Gyre mooring B. The waves may provide a source of energy for mixing within the deep interior Canada Basin. While mixing is difficult to quantify with the available data, there is some indication that density overturns may result from large vertical motions. Relatively little energy is required to cause significant overturns in the weakly stratified deep water. Further field measurements, ray-tracing and modeling studies are necessary, both to investigate the source and to understand the dynamical implications of these waves in the deep basin.

Acknowledgments. Funding was provided by the National Science Foundation Office of Polar Programs Arctic Sciences Section under awards ARC-0632201 and ARC-0806306. We appreciate the support of the captain and crew of the Canadian Coast Guard icebreaker, Louis S. St-Laurent, and we acknowledge financial and ship time support from Fisheries and Oceans Canada, the Canadian International Polar Year Program's Canada's Three Oceans project and by the U.S. National Science Foundation's Beaufort Gyre Exploration Project and collaboration with the Japan Agency for MarineEarth Science and Technology. We would like to thank Rick Krishfield for his support and efforts deploying and recovering the deep instruments on the mooring. Wavelet software was provided by C. Torrence and G. Compo (http://paos.colorado.edu/research/wavelets/). We thank two anonymous reviewers for invaluable comments.

\section{REFERENCES}

Brink, K. 1991. Coastal-trapped waves and wind-driven currents over the continental shelf. Annu. Rev. Fluid Mech., 23, 389-412.

Galt, J. 1973. A numerical investigation of Arctic Ocean dynamics. J. Phys. Oceanogr., 3, 379-396. Hamilton, P. 1990. Deep currents in the Gulf of Mexico. J. Phys. Oceanogr., 20, 1087-1104. -2009. Topographic Rossby waves in the Gulf of Mexico. Progr. Oceanogr., 82, 1-31.

Hogg, N. 2000. Low-frequency variability on the western flanks of the Grand Banks. J. Mar. Res., $58,523-545$.

Kelley, D. 1988. Explaining effective diffusivities within diffusive oceanic staircases, in Small-Scale Turbulence and Mixing in the Ocean, J. C. J. Nihoul and B. M. Jamart, eds., Elsevier, 541 pp.

Kelley, D., H. Fernando, A. Gargett, J. Tanny, and E. Özsoy. 2003. The diffusive regime of doublediffusive convection. Progr. Oceanogr., 56, 461-481. 
Pickart, R. 1995. Gulf Stream-generated topographic Rossby waves. J. Phys. Oceanogr., 25, 574-586.

Pickart, R., G. Moore, D. Torres, P. Fratantoni, R. Goldsmith, and J. Yang. 2009. Upwelling on the continental slope of the Alaskan Beaufort Sea: Storms, ice, and oceanographic response. J. Geophys. Res., 114, doi: 10.1029/2208JC005009.

Proshutinsky, A., R. Krishfield, M.-L. Timmermans, J. Toole, E. Carmack, F. McLaughlin, W. J. Williams, S. Zimmermann, M. Itoh, and K. Shimada. 2009. Beaufort Gyre freshwater reservoir: State and variability from observations. J. Geophys. Res., 114, doi: 10.1029/2008JC005104.

Reid, R. and O. Wang. 2004. Bottom-trapped Rossby waves in an exponentially stratified ocean. J. Phys. Oceanogr., 34, 961-967.

Rhines, P. 1970. Edge-, bottom-, and Rossby waves in a rotating stratified fluid. Geophys. Fluid Dyn., 1, 273-302.

Schlosser, P., K. Bernd, B. Ekwurzel, G. Bönisch, A. McNichol, R. Schneider, K. von Reden, H. Östlund, and J. Swift. 1997. The first trans-arctic C14 section: comparison of the mean ages of the deep waters in the Eurasian and Canadian basins of the Arctic Ocean. Nuclear Instruments and Methods in Physics Research Section B: Beam Interactions with Materials and Atoms, 123(1-4), 431-437.

Thompson, R. 1977. Observations of Rossby waves near site D. Progr. Oceanogr., 7, 135-162.

Thompson, R. and J. Luyten. 1976. Evidence for bottom-trapped topographic Rossby waves from single moorings. Deep-Sea Res., 1, 629-635.

Timmermans, M.-L., C. Garrett, and E. Carmack. 2003. The thermohaline structure and evolution of the deep waters in the Canada Basin, Arctic Ocean. Deep-Sea Res. I, 50, 1305-1321.

Timmermans, M.-L., H. Melling, and L. Rainville. 2007. Dynamics in the deep Canada Basin, Arctic Ocean, inferred by thermistor-chain time series. J. Phys. Oceanogr., 37, 1066-1076.

Timmermans, M.-L., J. Toole, R. Krishfield, and P. Winsor. 2008. Ice-tethered profiler observations of the double-diffusive staircase in the Canada Basin thermocline. J. Geophys. Res., 113, doi:10.1029/2008JC004829.

Torrence, C. and G. Compo. 1998. A practical guide to wavelet analysis. Bull. Am. Met. Soc., 79, 61-78.

Veronis, G. 1966. Rossby waves with bottom topography. J. Mar. Res., 24, 338-348.

Voinov, G. and E. A. Zakharchuk. 1999. Large-scale variations of sea level in the Laptev Sea, in Land-Ocean Systems in the Siberian Arctic: Dynamics and History, H. Kassens, H. A. Bauch, I. Dmitrenko, H. Eicken, and H.-W. Hubberten, eds., Springer-Verlag, 711 pp.

Zakharchuk, E. 2009. Anemobaric low-frequency waves in the Chukchi Sea. Russian Meteorology and Hydrology, 34, 56-67.

Received: 10 November, 2009; revised: 11 March, 2010. 